\title{
Shrinkage of the Entorhinal Cortex over Five Years Predicts Memory Performance in Healthy Adults
}

\author{
Karen M. Rodrigue and Naftali Raz \\ Institute of Gerontology and Department of Psychology, Wayne State University, Detroit, Michigan 48202
}

Lesions in the hippocampus (HC), the entorhinal cortex (EC), and the prefrontal cortex (PFC) are associated with impairment of episodic memory; reduced HC volume is linked to memory declines in dementia; and decline in EC volume predicts progression from mild cognitive impairment to dementia. However, in healthy adults, the relationship between memory and regional volumes is unclear, and no data are available on the relationship of longitudinal regional shrinkage to memory performance in a cognitively intact population. The objective of this study was to examine whether shrinkage of the EC, HC, and PFC over a 5 year period can predict declarative memory performance in healthy adults. The volumes of three brain regions were measured on magnetic resonance images that were acquired twice, 5 years apart. Multiple measures of episodic memory were administered at follow-up. Results indicated that the volume of $\mathrm{HC}$ and PFC (but not EC) correlated with age at baseline and follow-up. However, after age differences in memory were taken into account, none of the regional volumes was associated with memory performance at follow-up. In contrast, greater annual rate of shrinkage in EC (but not $\mathrm{HC}$ or PFC) predicted poorer memory performance. Thus, in a healthy and educated population, even mild age-related shrinkage of the EC may be a sensitive predictor of memory decline.

Key words: magnetic resonance imaging; aging; volumetry; longitudinal studies; memory; medial temporal lobe; hippocampus; prefrontal cortex

\section{Introduction}

Age-related declines in episodic memory are common (Verhaeghen et al., 1993), but their neuroanatomical underpinnings are unclear. Aging differentially affects the putative brain substrates of memory: the hippocampus (HC), the entorhinal cortex (EC), and the prefrontal cortex (PFC) (Squire and Zola-Morgan, 1991; Buckner, 2003). The PFC appears to be the most vulnerable of the three, whereas the effect of aging on the HC is moderate, and the EC is relatively spared (Raz, 2000; Du et al., 2003; Raz et al., 2004a). In contrast, in the course of Alzheimer's disease (AD), pathological changes in EC and $\mathrm{HC}$ precede those in the neocortex (Hyman et al., 1984; Braak and Braak, 1991; Laakso, 2002; Thompson et al., 2003). Although shrinkage of both the HC (Xu et al., 2000) and EC (Dickerson et al., 2001) is associated with concurrent $\mathrm{AD}$ and predicts clinical deterioration (Jack et al., 2000), volume reduction in the EC is viewed as the earliest indicator of incipient conversion from preclinical cognitive impairment to dementia (Killiany et al., 2000; Dickerson et al., 2001).

Although it is plausible that age-related memory declines stem from deterioration of the PFC and the medial temporal structures, the connection between weakening memory and PFC volume has never been proven, and the association between shrinkage of the $\mathrm{EC}$ or $\mathrm{HC}$ and declines in memory has been replicated only in pathological samples (Wilson et al., 1996; Convit et al.,

Received Sept. 11, 2003; revised Nov. 30, 2003; accepted Dec. 2, 2003.

Correspondence should be addressed to Dr. Naftali Raz, Institute of Gerontology, Wayne State University, 87 East Ferry Street, Detroit, MI 48202. E-mail: nraz@wayne.edu.

DOI:10.1523/JNEUROSCI.4166-03.2004

Copyright $\odot 2004$ Society for Neuroscience $\quad$ 0270-6474/04/240956-08\$15.00/0
1997), in which volumetric shrinkage has been linked to neuronal loss (Bobinski et al., 2000; Kordower et al., 2001). In vivo studies of association between memory and $\mathrm{HC}$ volume in healthy adults have produced contradictory, sometimes diametrically opposite, results (Golomb et al., 1994; Soininen et al., 1994; Sullivan et al., 1995; Raz et al., 1998; Du et al., 2003). As for EC, to date only two in vivo studies have linked its shrinkage to declines in memory performance in adults who were at increased risk for developing dementia (de Toledo-Morrell et al., 2000), and to septuagenarians and octogenarians (Du et al., 2003).

The latter studies have several shortcomings. First, the samples were limited to people with cognitive risk factors or the very old. Second, in those studies, neither the HC nor the PFC was considered to be a comparison region of interest (ROI), leaving the question of specificity open. Because memory differences are apparent in cognitively intact persons of younger age, and because memory declines may reflect changes in multiple brain sites, it was important to address these limitations. The main objective of this study was to examine whether longitudinal changes and cross-sectional age differences in the PFC, EC, and HC volumes predict episodic memory performance in a wide age range, and whether the predictive ability differs across the regions. To attain those objectives, we measured PFC, EC, and HC volumes in a sample of healthy adults on two occasions, 5 years apart. At follow-up, we also assessed episodic memory with various test formats, immediate and delayed.

\section{Materials and Methods \\ Participants}

The data for this study were collected in an ongoing investigation of neuroanatomical correlates of age-related differences in cognition, con- 
ducted in a major metropolitan area in the United States. Participants of previous cross-sectional studies, who were initially recruited by advertising in local media and on the university campus, were contacted by mail and telephone for longitudinal follow-up. We attempted to locate 284 participants who were eligible for a second scan after a 5 year delay, and 95 of them completed the study. At the follow-up, the data were collected on all of the eligible participants. However, the same health screening criteria were applied to the follow-up sample as were used to determine eligibility at the baseline (time 1), and participants whose health status changed for the worse were not included in this study.

The participants signed a consent form approved both by the University Committee for Protection of Human Subjects in Research and by the hospital's Patients Participation Committee. All of the participants were screened using a health questionnaire completed by the participants and augmented with telephone and personal interviews. Persons who reported history of cardiovascular, neurological, or psychiatric conditions, head trauma with loss of consciousness for more than $5 \mathrm{~min}$, thyroid problems, diabetes, treatment for drug and alcohol problems, or a habit of taking more than three alcoholic drinks per day were excluded from the study. None of the participants used antiseizure medication, anxiolytics, or antidepressants. Persons who suffered or suspected they could suffer from claustrophobia were advised against participation in the study.

All of the subjects were screened for dementia and depression using a modified Blessed Information-Memory-Concentration Test (BIMC) (Blessed et al., 1968) with a cutoff of $85 \%$ correct, Mini-Mental State Examination (MMSE) (Folstein et al., 1975) with a cutoff of $26(87 \%$ correct), and Geriatric Depression Questionnaire (CES-D) (Radloff, 1977) with a cutoff of 15 . The BIMC and CES-D were administered on both testing occasions, whereas MMSE was used only at follow-up (time 2). An experienced neuroradiologist examined the magnetic resonance (MR) scans for signs of space-occupying lesions and signs of significant cerebrovascular disease. All of the participants were consistent righthanders, as indicated by a score of $>75 \%$ on the Edinburgh Handedness Questionnaire (Oldfield, 1971).

Although 95 subjects completed the follow-up, 19 were excluded from data analyses, because they did not meet the health criteria. The subjects (age, 46-83 years) were excluded because of Parkinson disease (1), cerebral hemorrhage (2), cardiac bypass surgery (4), angioplasty (1), newly diagnosed hypothyroid (3), diabetes mellitus (4), and cancer (4). The follow-up data on an additional 22 subjects could not be used in the longitudinal study because of a technician's error in setting an acquisition parameter (field of view), which would produce a confound for time of measurement and scan resolution. Although loss of those data reduced statistical power, it did not introduce any detectable bias, for those were consecutive admissions to the study, and were not selected by any specific criteria. Thus, the remaining sample consisted of 54 subjects. Volumetric measures on these participants have been reported previously (Raz et al., 2003a,b, 2004a). Six participants did not have the majority of the cognitive data, and the remaining group ( $n=48 ; 19$ men and 29 women) comprised the sample for the current study. The average duration of the follow-up delay was $5.20( \pm 0.22)$ years, with a range between 5.00 and 5.92 years. There was no difference between men and women in the duration of the follow-up delay ( 5.18 years for women vs 5.25 years for men). The age of the participants at follow-up (time 2) ranged between 26 and 82 years, and the mean was $57.62( \pm 15.20)$ years. Men were somewhat younger than women (men, 52.60 years; women, 61.21 years; $t=1.88 ; p=0.06$ ). The average level of education in this sample was high (15.96 \pm 2.33 years of formal schooling) and did not differ between the sexes: 15.72 years for women versus 16.32 years for men $(t<1$; NS). Although an MMSE score of 26 was used as a cutoff to exclude subjects with dementia, no healthy participants obtained that score at follow-up, and the MMSE scores were higher than the cutoff $(29.06 \pm 0.93)$. The scores were unrelated to age $(r=-0.13$; NS) or sex $(t=1.07$; NS). Blessed scores did not change in 5 years: $t<1$ (NS).

By the time of follow-up, 16 participants ( 5 men and 11 women; mean age, $60.81 \pm 12.70$ years) carried a diagnosis of hypertension and were taking antihypertensive medications. The hypertensive participants were older than the remainder of the sample $(t=3.04 ; p<0.01)$ but had the same number of years of formal schooling and equivalent MMSE scores (both $t<1$; NS).

\section{MR imaging protocol}

All of the imaging was performed on $1.5 \mathrm{~T}$ Signa scanners (General Electric, Milwaukee, WI) installed in the same hospital. However, whereas only one magnet was used at baseline, one follow-up scan was acquired on an identical scanner located in an adjacent room, and two participants were rescanned on a mobile 1.5 T General Electric magnet located at the entrance of the imaging center. The vast majority of the participants (93\%) were rescanned on the same scanner at follow-up. The imaging site was fully staffed by medical physicists and MR imaging technologists, and the scanners were routinely calibrated using a standard General Electric phantom.

Sagittal localizer images with repetition time (TR) of $400 \mathrm{msec}$, echo time (TE) of $16 \mathrm{msec}$, one excitation, and slice thickness of $5.0 \mathrm{~mm}$ were acquired first. A fast spin echo (FSE) sequence of dual-echo $\mathrm{T}_{2}$ - and proton density-weighted axial images (TR/TE, 3300/90ef or 18ef/1; slice thickness, $5 \mathrm{~mm}$; interslice gap, $2.5 \mathrm{~mm}$ ) was acquired to screen for age-related cerebrovascular disease. Volumes were measured on two sets of images acquired using a $\mathrm{T}_{1}$-weighted three-dimensional spoiled gradient recalled sequence with 124 contiguous axial slices, TE of $5 \mathrm{msec}$, TR of $24 \mathrm{msec}$, field of view of $22 \mathrm{~cm}$, acquisition matrix of $256 \times 192$, slice thickness of $1.3 \mathrm{~mm}$, and flip angle of $30^{\circ}$.

\section{Volumetric image analysis}

\section{$M R$ image processing}

The images were reformatted to correct for undesirable effects of head tilt, pitch, and rotation, using BrainImage 2.3.3 software available online at http://www.stanford.edu/group/cap/research/neuroimaging/imageanalysis. The process [described in detail previously by Raz et al. (2004b)] consisted of aligning the axial plane to pass through the anterior and posterior commissures (incorporating the anterior commissure-posterior commissure line), adjusting the sagittal plane to pass along the straight line drawn through the extreme anterior and posterior points of the interhemispheric fissure, and aligning the coronal plane perpendicular to the axial and sagittal planes as defined above. Reformatted images were partitioned into contiguous $1.5 \mathrm{~mm}$ coronal sections.

The ROI areas were measured with NIH Image (version 1.60; available online at http://rsb.info.nih.gov/nih-image/). The slices that comprised any given ROI were divided into two equal groups at random, and each half-sample was traced by a different operator. The images acquired on both occasions (time 1 or 2) were coded, and the order of tracing was randomized within each subject by a person other than the operators who traced the ROIs. The operators were blind to the chronology of the specific images, and the demographic and cognitive characteristics of the participants, as well as to the magnet on which the scan was acquired. Volumes were computed by multiplying the sum of the areas by slice thickness and by the calibration factor derived from phantom measurements.

Reliability of manually traced ROI volumes [examples in Raz et al. (2004a,b)] was assessed using intraclass correlation (ICC [2]; random raters formula) (Shrout and Fleiss, 1979). The reliabilities were 0.99 for the intracranial volume (ICV), 0.96 for the lateral PFC (LPFC), 0.93 for the $\mathrm{HC}$, and 0.92 for the EC.

\section{Region demarcation and tracing rules \\ Intracranial volume}

The intracranial volume was estimated from the coronal sections. The operator traced ICV on every eighth slice between the first slice following the orbits and last slice on which brain tissue was visible. Tracing began at the right side of the head and proceeded clockwise following the inner table of the skull until the same boundary was reached on the left side of the head, at which point the tracing contour was allowed to close automatically by drawing a straight line back to the origin.

\section{Hippocampus}

The HC volume was measured on a series of coronal slices aligned perpendicular to the long axis of the right hippocampus. The mammillary 
bodies defined the rostral boundary of the $\mathrm{HC}$, and the slice showing the fornices rising from the fimbria marked its caudal boundary. Care was taken not to include the amygdala in this ROI.

\section{Entorhinal cortex}

In tracing this region, we followed the rules developed by Insausti et al. (1998) with minor modifications. The EC was traced on consecutive slices beginning two slices posterior to the limen insula and continuing to one slice caudal to the posterior limit of the gyrus intralimbicus. Rather than defining the ventrolateral boundary by estimation of the depth of the collateral sulcus, the medial bank of the collateral sulcus was used in all of the cases. The modification is on the conservative side, so as to avoid overestimation of the EC.

\section{Lateral prefrontal cortex}

Eight to 12 coronal slices located within $40 \%$ of the distance between the genu of the corpus callosum and the frontal pole were included in the prefrontal ROI. The LPFC ROI included superior, middle, and inferior frontal gyri and covered primarily Brodmann areas 9 and 46, and parts of areas 8,10 , and 45 . The superior and inferior boundaries of the LPFC were the most dorsomedial point of the cortex and the lateral orbital sulcus, respectively.

\section{Cognitive instruments and procedures}

The following cognitive tasks were administered to each participant individually by trained technicians. Because participants were pooled from three different cognitive studies, the order of task administration varied depending on the schedule of the particular study.

\section{Association memory tests}

Memory for Names Immediate. This auditory-visual paired-associate task is a subtest from Woodcock-Johnson Psychoeducational Battery, Revised (Woodcock and Johnson, 1989). During acquisition, the participant is asked to learn associations between an imaginary space creature and the creature's name. At test, the participant is shown a target creature and an array of nine additional creatures, and is asked to point to the target creature that the experimenter names. During this acquisition phase, corrections are given to incorrect responses. The level of difficulty increases progressively as the participants are required to remember the names of more space creatures. The index of interest is the total number of correctly identified creatures. The reliability of this test is $r=0.91$ (Woodcock and Mather, 1989).

Paired-Associate Learning Immediate. This is a subtest from the Wechsler Memory Scale, Revised (Wechsler, 1987). In each trial of the learning phase, participants hear eight pairs of words (four pairs with high- and four with low-association value). Immediately after each reading of the list, participants are presented with a one-word cue from each pair and asked to recall the second word of the pair. Participants who fail to complete all of the pairs correctly by the third trial are given up to six additional trials until all eight targets are correctly remembered. The index of performance for all of the participants is the total number of words correctly recalled in the first three sets of trials. The test-retest reliability of immediate Paired Associates Learning (PAL)-Immediate is $r=0.60$.

\section{Free-recall tests}

Logical Memory Immediate. This is a subtest from the Wechsler Memory Scale, Revised (Wechsler, 1987). During acquisition, participants hear a short story and are immediately asked to repeat as much of the story as they remember. This procedure is repeated with a second short story. The score is the number of correctly recalled pieces of information from both stories. The test-retest reliability of this test is $r=0.74$.

California Verbal Learning Test. The California Verbal Learning Test (CVLT) (Psychological Corporation, San Antonio, TX) is a standard, widely used measure of memory. It produces several indices, including recognition, free and cued recall, both immediate and delayed. Although useful in clinical neuropsychological assessment, the multiple CVLT indices are highly correlated and are confounded by the effects of order of measurement. For the purposes of this study, we chose the sum of items correctly recalled from list A, trials $1-5$, as an index of immediate mem- ory. Because of the repeated presentation and recollection of the list A stimuli across the test, in addition to the presentation of list B, no pure measure of delayed recall can be derived from the CVLT.

Delayed-memory measures. The same three tasks [Logical Memory Immediate (LM), Memory for Names Immediate (MFN), and PAL] were administered again after a 20 min delay. At the initial test phase, participants are told that both the LM and the PAL tests will be given again, whereas no warning is given at the initial MFN administration. The reliabilities for the delayed tests are as follows: LM, $r=0.75$; MFN, $r=$ 0.91 ; and PAL, $r=0.41$ (likely because of restriction of score range).

Because the participants were recruited initially for various studies, only 28 ( 14 women) of 48 had partial baseline memory measurements (CVLT, LM, and PAL; none had MFN). Those participants did not differ from the rest of the sample in age (52 years), education (16 years), or MMSE scores (29).

\section{Data conditioning and analytical approach}

The dilemma facing investigators in the case of multiple measures of similar constructs (i.e., memory) is whether to analyze each index separately, because they may measure distinct behavioral and cognitive expressions of the construct, or to amalgamate the test scores into a composite index, thus losing some of the specificity but gaining reliability and statistical power. The presence of multiple correlated indices of a construct in a linear model produces multicollinearity, which reduces reliability of regression coefficients and harms the validity of inference. Pearson correlations among the immediate-memory tests ranged from $r=$ 0.49 to 0.61 (all of the values of $p<0.01$ ); for delayed-memory tests, the correlations ranged from $r=0.48$ to 0.51 (all of the values of $p<0.01$ ). Thus, the correlations were sufficiently high to indicate that if the variables were entered simultaneously into a multiple regression model, multicollinearity would ensue. Consequently, all of the raw memory scores were standardized and averaged to create four composite scores: association memory and free recall, immediate and delayed. Recall composite correlated $r=0.55$ and 0.66 , both $p<0.001$ with association memory composite for immediate and delayed tests, respectively, and the correlation between the immediate- and the delayed-memory composites was very high $(r=0.86 ; p<0.001)$. Nonetheless, we retained the separate composites for analyses under the theoretical assumptions that two types of memory functions tested under two different conditions may be driven by distinct neural processes. Separate models were constructed to predict delayed- and immediate-memory performance for association memory and free recall.

Because this sample was not selected on memory-based criteria, we checked whether any of the participants fit the description of mild cognitive impairment (MCI). Definitions of MCI abound (Palmer et al., 2003), and we chose the most commonly used Mayo Clinic definition (Petersen et al., 1999), which emphasizes isolated memory declines of 1.5 $\mathrm{SD}$ below the age-adjusted mean. Examination of memory test scores in our sample reveals no participants who fit the MCI criteria. Even among the oldest 10 participants (age, >70), the MMSE scores were within the range of 27-30 with a mean of 28.8, and Logical Memory scores were substantially above those of the normal controls in the Mayo sample (26 for the immediate and 21 for the delayed recall; age-adjusted percentile scores ranging from 29 to $75 \%$ ). No scores on CVLT and WoodcockJohnson (WJ) Memory for Names deviated from the norms by 1.5 SD.

Brain measures also required preanalysis conditioning. Because men generally have significantly larger body size than women do, measured brain volumes must be adjusted accordingly. In addition, men in this sample were younger than women, which, in combination with sex differences in body size, could confound age and sex differences in brain volumes. The ICV differed between the sexes $(t=-4.57 ; p<0.001)$ and correlated with height $(r=0.57$ at baseline and $r=0.65$ at follow-up; both $p<0.001)$. However, it did not correlate with age $(r=-0.13$ on both occasions; NS), and remained stable across the 5 year delay $(F<1)$. Thus, we used ICV to adjust the regional volumes via the analysis of covariance formula: adjusted volume $=$ raw volume $-b \times(\mathrm{ICV}-$ mean $\mathrm{ICV}$ ), where $b$ is the slope of regression of the appropriate ROI volume on ICV. In all of the analyses presented below, we used the ICV-adjusted volumes. 
Table 1. Descriptive statistics for brain and memory measures

\begin{tabular}{|c|c|c|c|c|c|c|c|c|}
\hline \multirow[b]{2}{*}{ Variables } & \multicolumn{4}{|c|}{ Baseline } & \multicolumn{4}{|c|}{ Follow-up } \\
\hline & Mean & SD & $\mathrm{CV}$ & $r_{\text {age }}$ & Mean & SD & CV & $r_{\text {age }}$ \\
\hline \multicolumn{9}{|l|}{ Brain } \\
\hline Left hippocampus & 3.25 & 0.37 & 0.11 & $-0.42^{* *}$ & 3.11 & 0.39 & 0.13 & $-0.54^{* * *}$ \\
\hline Right hippocampus & 3.23 & 0.38 & 0.12 & $-0.36^{*}$ & 3.08 & 0.40 & 0.13 & $-0.46^{* * *}$ \\
\hline Total hippocampus & 6.48 & 0.71 & 0.11 & $-0.42^{* *}$ & 6.19 & 0.76 & 0.12 & $-0.53^{* * *}$ \\
\hline Left entorhinal cortex & 1.32 & 0.16 & 0.12 & -0.04 & 1.29 & 0.16 & 0.12 & -0.10 \\
\hline Right entorhinal cortex & 1.32 & 0.16 & 0.12 & -0.04 & 1.29 & 0.17 & 0.14 & -0.01 \\
\hline Total entorhinal cortex & 2.63 & 0.30 & 0.11 & -0.04 & 2.58 & 0.32 & 0.12 & -0.04 \\
\hline Left lateral prefrontal cortex & 9.01 & 1.03 & 0.12 & $-0.58^{* * *}$ & 8.57 & 1.15 & 0.13 & $-0.56^{* * *}$ \\
\hline Right lateral prefrontal cortex & 9.26 & 1.24 & 0.13 & $-0.52^{* * *}$ & 8.80 & 1.36 & 0.15 & $-0.60^{* * *}$ \\
\hline Total lateral prefrontal cortex & 18.27 & 2.12 & 0.12 & $-0.57^{* * *}$ & 17.38 & 2.42 & 0.14 & $-0.60^{* * *}$ \\
\hline \multicolumn{9}{|l|}{ Memory } \\
\hline Logical memory immediate & & & & & 29.35 & 5.85 & 0.20 & $-0.33^{*}$ \\
\hline Logical memory delayed & & & & & 25.27 & 6.63 & 0.26 & $-0.39^{* *}$ \\
\hline WJ memory for names immediate & & & & & 55.94 & 11.15 & 0.20 & $-0.53^{* * *}$ \\
\hline WJ memory for names delayed & & & & & 23.96 & 8.62 & 0.36 & $-0.58^{* * *}$ \\
\hline Paired associates immediate recall & & & & & 19.00 & 2.87 & 0.15 & $-0.32^{*}$ \\
\hline Paired associates delayed recall & & & & & 7.35 & 1.00 & 0.14 & $-0.37^{* *}$ \\
\hline CVLT immediate list recall (trials 1-5) & & & & & 56.63 & 10.04 & 0.18 & $-0.53^{* * *}$ \\
\hline
\end{tabular}

Volumes are in cubic centimeters and are adjusted for cranial volume. CV, Coefficient of variation. ${ }^{*} p<0.05 ;{ }^{* *} p<0.01 ;{ }^{* * *} p<0.001$.

A mixed general linear model analytic framework was used in the analyses presented below. All of the nonsignificant interactions were removed from the final model. To control for the inflated significance levels attributable to violation of sphericity assumption of the repeatedmeasures analysis, we used the Huynh-Feldt correction factor. Before analyzing the data, we examined the distributions for outliers and gross deviations from normality.

\section{Results}

Descriptive statistics for all of the memory tasks and brain variables are presented in Table 1 . The associations between age and regional volumes follow the pattern observed in previous reports, although the correlation between age and $\mathrm{HC}$ volume (especially at follow-up) is somewhat larger than the reported median (for review, see Raz, 2000). The magnitude of age-related memory differences are in the typical range reported in the literature (Verhaeghen et al., 1993).

\section{The effects of age, regional volume change, and sex on memory}

Zero-order correlations between the brain volumes and composite memory scores revealed no links between EC volume and memory composites ( $r=-0.01$ to 0.23 ; all NS). The HC volume showed somewhat stronger links with memory, although only one correlation of eight reached significance: $r=0.00$ to 0.30 , all NS, except for the follow-up volume and associative immediate recall: $r=0.33$ and $p<0.05$. The volume of the lateral prefrontal cortex correlated significantly with both memory measures: $r=$ $0.23-0.35$, with five of eight correlations significant at $p<0.05$. However, all of the memory composites and regional volumes of LPFC and HC significantly correlated with age, indicating a necessity to examine the link between local volumes and memory in the context of age-related differences.

To determine the unique influence of age and regional brain volumes on memory performance, we submitted the data to a mixed linear model with age, LPFC, HC, and EC volumes at follow-up (i.e., concurrent with memory measures) as continuous independent variables, sex as a categorical independent variable, and composite memory indices (associative vs free recall, and immediate vs delayed) as two repeated measures. This analysis revealed a significant main effect of age $\left(F_{(1,42)}=9.97 ; p<\right.$
0.01). There was a significant interaction between hippocampal volume, test type, and delay duration: $F_{(1,42)}=4.49$ and $p<0.05$. This effect reflected the difference among correlations between HC volume and memory measures, with one of four (immediate associative recall) showing a significant link: $r=0.33$ and $p<$ 0.05 . No other significant effects of brain volumes on memory were noted (all main effects and interactions, except one, $F<1$; for EC volume effect, $F_{(1,42)}=1.39$; NS).

All three regional volumes shrunk over a 5 year period. The annualized rates of decline [annualized percent change (APC)], however, differed among the ROIs. The EC showed a significantly slower decline (APC, $0.37 \%$ ) than HC (APC, $0.85 \% ; t=2.90 ; p<$ 0.006 ) or PFC (APC, $0.96 \% ; t=3.48 ; p<0.001$ ), whereas no difference between PFC and HC was noted $(t=0.68$; NS). The relationships between age and regional shrinkage are illustrated in Figure 1, which shows a lack of dependence of entorhinal shrinkage on age and significant age-related acceleration of shrinkage in $\mathrm{HC}$ and PFC (both tests for quadratic component are significant, at $p<0.001$ for $\mathrm{HC}$ and $p<0.05$ level for PFC).

To examine the effects of age-related differences and regional brain changes on memory performance, we submitted both immediate- and delayed-memory composite indices to a linear model analysis as outlined above. In this analysis, the composite indices of memory were dependent measures (associative vs free recall, and delayed vs immediate). Age at baseline (centered at its sample mean) was a continuous independent variable, sex was a categorical independent variable, and annual rate of change in $\mathrm{PFC}, \mathrm{EC}$, and $\mathrm{HC}$ volumes served as continuous independent variables.

The analysis yielded a significant main effect of age $\left(F_{(1,42)}=\right.$ $13.72 ; p<0.001)$, but no age $\times$ memory index or age $\times$ memory type interaction $(F<1$; NS). Older adults attained lower memory test scores regardless of test type or delay duration (Table 1). No reliable effect of sex on memory performance was found, although a trend $\left(F_{(1,42)}=3.76 ; p<0.06\right)$ was evident, with women performing slightly better than men. No sex $\times$ memory index or sex $\times$ delay interaction was observed $\left(F_{(1,42)}=1.92\right.$ and $F<1$; both NS). Change in EC volume predicted memory performance $\left(F_{(1,42)}=6.01 ; p<0.05\right)$, whereas change in PFC and HC volumes failed to account for any unique variance beyond that ex- 


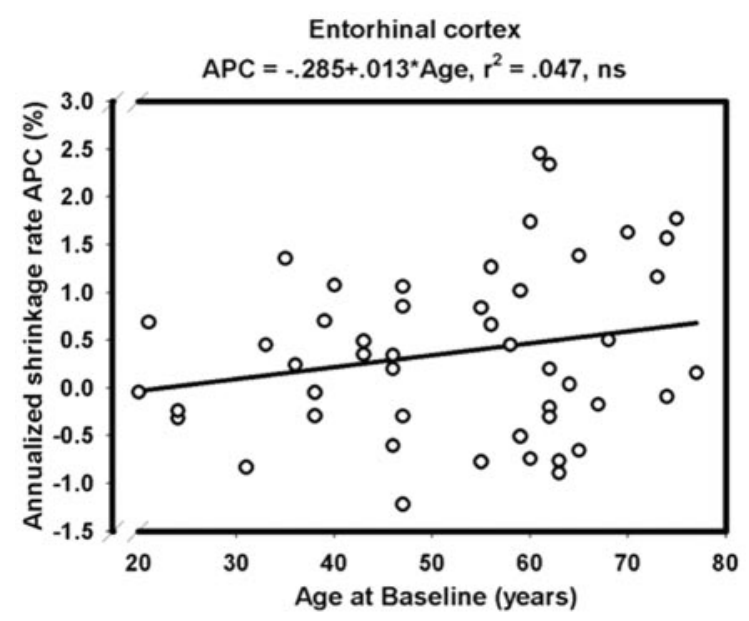

Prefrontal Cortex

APC $=3.024-.125^{\star}$ Age $^{-} .0015^{\star}$ Age $^{2}, r^{2}=.200, p<.01$

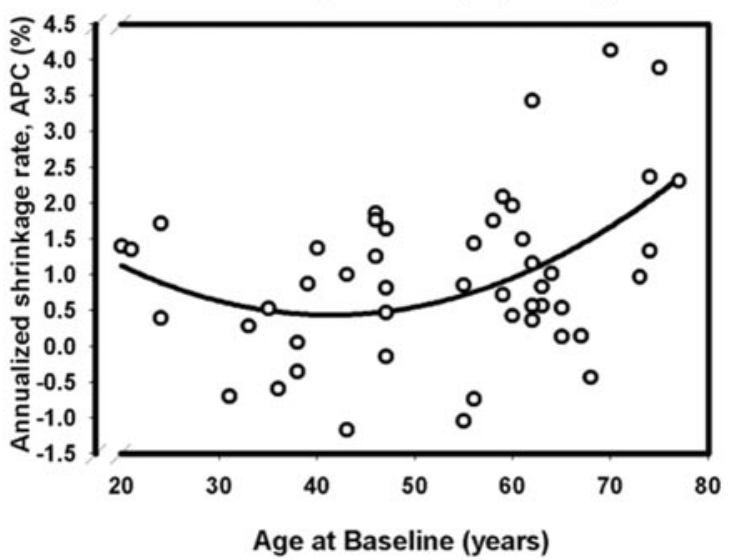

Hippocampus



Figure 1. Scatter plots of the associations between age and the regional shrinkage of three brain regions: $\mathrm{EC}, \mathrm{PFC}$, and $\mathrm{HC}$. Note the nonlinear relation of $\mathrm{PFC}$ and $\mathrm{HC}$ shrinkage rates with age, indicating acceleration of decline in older participants. The regression equations and the goodness-of-fit index $\left(r^{2}\right)$ are presented above each plot.

plained by the calendar age, $F<1$. That is, greater shrinkage of the EC volume over the 5 year lag predicted poorer memory performance at follow-up after accounting for the influence of age. In addition, no differential effect of longitudinal change in



Figure 2. A scatter plot and regression of delayed-memory composite score $(z)$ on age. Larger $z$ represents better memory performance. The regression equation describing the relationship between the two variables and the goodness-of-fit index $\left(r^{2}\right)$ are presented above the plot.

EC volume was evident on immediate versus delayed memory, or associative versus free recall, as indicated by a lack of significant EC $\times$ memory index and EC $\times$ delay interaction, $F<1$. Neither ROI $\times$ sex nor ROI $\times$ age interaction was reliable (values of $F<$ 1). Adding a quadratic age component to the equation did not alter the results (all of the values of $F<1$ ).

Because of a high correlation among the immediate and delayed indices for associative and free recall and similarity of their behavior in the linear models, we present the results only for combined delayed-memory index. In Figures 2 and 3, we present regressions of memory performance on age and regional volumes. The plots and regression equations show clear age dependence of the memory composite and a significant link between shrinkage and memory only for the EC. In sum, the oldest participants with the greatest EC shrinkage demonstrated the worst memory.

If one presumes that positive change (growth) of a cortical volume is an error, an artificial expansion of the shrinkage range by scores that are essentially errors would be a cause for concern. Such range expansion could have inflated the association between the index of shrinkage and the criterion variable. To examine whether such influence was introduced in our sample, we analyzed the data with only participants who showed shrinkage or stability ( $\mathrm{APC} \geq 0$ ) of the regional volumes. Although that manipulation resulted in the loss of statistical power, the results did not change.

Because only partial data were available for the baseline memory measures, we did not have sufficient power to examine the possible associations between shrinkage of the brain regions and longitudinal decline in memory performance. With only 28 subjects, we found that only 1 of 15 possible correlations between three regional indices of shrinkage and five tests (LM, LM delayed, PAL, PAL delayed, and CVLT) reached significance. It was the correlation between HC shrinkage and LM immediate score decline: $r=0.50, p<0.01$, uncorrected. Such a finding would be expected by chance.

Previous research indicates that a history of hypertension can enhance age-related differences in regional cortical volumes and can negatively affect memory and control processes (Strassburger et al., 1997; Waldstein and Katzel, 2001; Raz et al., 2003c), There- 



Annualized shrinkage, APC (\%)

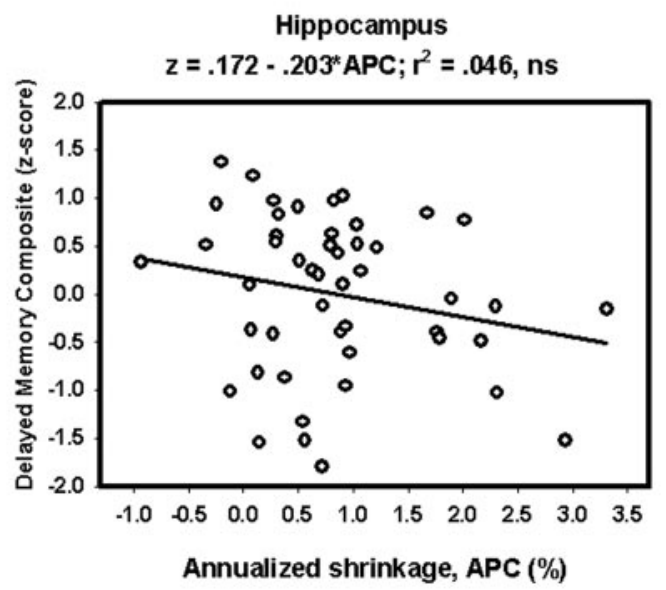

Figure 3. Scatter plots and regressions describing the associations between regional shrinkage rates (APC) and memory performance ( $z$ ) for three regions of interest (EC, PFC, and $\mathrm{HC}$ ). Regression equations describing the relations and the goodness-of-fit index $\left(r^{2}\right)$ are presented above each plot. Note that, with removal of an outlier (65-year-old man), the association between entorhinal shrinkage rate and delayed-recall composite score $(z)$ was strengthened: $r^{2}=0.235$ and $p<0.001$.

fore, we repeated all of the analyses after removing from the sample the 16 participants ( 5 men and 11 women; age range, 38-77 years) who reported having medically controlled hypertension. Because the results remain unchanged, we decided to retain those participants in the sample.

\section{Discussion}

The main finding in this study is that, in healthy and educated elderly, longitudinal shrinkage of the entorhinal cortex is associated with reduced memory performance. Negative change in EC volume predicted both immediate- and delayed-memory performance in free recall and association memory domains above and beyond the effects of calendar age. In contrast, after accounting for age-related effects, memory performance was unrelated to longitudinal change in PFC and HC volume. The observed differences are not artifacts of differential reliability of the measures, because PFC, EC, and $\mathrm{HC}$ volume estimates were equally reliable and stable over time. Although the EC and HC shrinkage in this sample have been described previously (Raz et al., 2004a), the longitudinal shrinkage of the prefrontal cortex is a new finding, consistent with previously reported longitudinal estimates in healthy adults (Pfefferbaum et al., 1998; Resnick et al., 2003).

The observed link between EC shrinkage and memory that was independent of age across a wide age span augments the findings in the very old (Du et al., 2003) and memory-impaired (de Toledo-Morrell et al., 2000) adults. However, in contrast to de Toledo-Morell and her colleagues, we found no differential effect of volume on delayed- versus immediate-memory measures. One reason for this discrepancy may be the use of a shorter delay (20 $\mathrm{min}$ instead of $40 \mathrm{~min}$ ) in the present study and the ensuing minimization of the distinction between immediate and delayed recall. Another reason may be the differences in sample composition: de Toledo-Morrell et al. (2000) focused on identifying predictors of incipient dementia and recruited normal adults who presented with memory complaints. Those participants were more impaired than the participants in the present sample of healthy volunteers and, as a result, exhibited greater delayed-memory deficits as would be expected in early dementia (Laakso et al., 2000).

The mechanisms of the observed association between EC shrinkage and memory are unclear, because little is known about the neural and histological correlates of the observed volume declines. However, a recent examination of the brains whose donors' memory was assessed antemortem (Guillozet et al., 2003) provides interesting clues. In that study, a strong negative association was found between the density of neurofibrillary tangles in the layer II of the EC and word list recall. The donors in that study were free of dementia as established by serial neuropsychological and neurological examinations. However, some brains in that small sample belonged to individuals who were characterized as mildly cognitively impaired and who displayed distinctly high tangle density and distinctly poor memory performance. Thus, it is possible that neural processes signaled by increased density of neurofibrillary deformations are responsible for gross volume decline of the EC and are accompanied by disruption of vital memory circuits. Because regional volumes were not reported by Guillozet et al. (2003), it is unknown whether the hypothesized relationship existed in their sample.

We report the observations of the present study in a sample characterized by high education and very good physical and cognitive health that was followed for 5 years in uniform manner. The advantage of longitudinal designs is the minimization of individual differences and cohort effects that can confound age effects in cross-sectional studies. However, selective attrition in longitudinal studies tends to bias follow-up samples toward inclusion of individuals who are more cognitively stable overall than at baseline testing (Lindenberger et al., 2002). Thus, although generalizability of the findings is reduced, so is the likeli- 
hood of including individuals in prodromal stages of dementia. It should also be noted that generalizability of the present study is further limited, because the findings were obtained in a highly selective sample. Participants were highly educated, and care was taken to exclude individuals with a variety of common agerelated diseases, except for treated hypertension, which evidently had no impact on the observed pattern of results. Thus, our findings are likely to reflect successful, rather than typical aging.

In summary, age-related shrinkage of the entorhinal cortex in a wide age range of adults, however mild, appears to be sensitive to memory deficits in ostensibly healthy, nonimpaired adults. Unlike in bona fide dementia and amnesic syndromes, reduction of the HC volume is not a sensitive predictor of memory performance. We cannot rule out the possibility that the EC memory connection stems from developing yet clinically undetectable pathology. It is unclear how many, or if any, of the participants who evidenced EC shrinkage and lower memory scores but remained cognitively intact in a 5 year period are destined to develop dementia. This question can be answered only by an additional longitudinal follow-up.

\section{References}

Blessed G, Tomlinson BE, Roth M (1968) The association between quantitative measures of dementia and senile change in the cerebral grey matter of elderly subjects. Br J Psychiatry 114:797-811.

Bobinski M, de Leon MJ, Weigel J, De Santi S, Convit A, Saint LL, Rusinek H, Wisniewski HM (2000) The histological validation of post mortem magnetic resonance imaging-determined hippocampal volume in Alzheimer's disease. Neuroscience 95:721-725.

Braak H, Braak E (1991) Neuropathological stageing of Alzheimer-related changes. Acta Neuropathol (Berl) 82:239-259.

Buckner R (2003) Functional-anatomic correlates of control processes in memory. J Neurosci 23:3999-4004.

Convit A, de Leon MJ, Tarshish C, De Santi S, Tsui W, Ruisnek H, George A (1997) Specific hippocampal volume reductions in individuals at risk for Alzheimer's disease. Neurobiol Aging 18:155-158.

de Toledo-Morrell L, Goncharova I, Dickerson B, Wilson RS, Bennett DA (2000) From healthy aging to early Alzheimer's disease: in vivo detection of entorhinal cortex atrophy. Ann NY Acad Sci 911:240-253.

Dickerson BC, Goncharova I, Sullivan MP, Forchetti C, Wilson R, Bennett DA, Beckett LA, de Toledo-Morrell L (2001) MRI-derived entorhinal and hippocampal atrophy in incipient and very mild Alzheimer's disease. Neurobiol Aging 22:747-754.

Du AT, Schuff N, Zhu XP, Jagust WJ, Miller BL, Reed BR, Kramer JH Mungas D, Yaffe K, Chui HC, Weiner MW (2003) Atrophy rates of entorhinal cortex in AD and normal aging. Neurology 60:481-486.

Folstein MF, Folstein SE, McHugh PR (1975) "Mini-mental state." A practical method for grading the cognitive state of patients for the clinician. J Psychiatr Res 12:189-198.

Golomb J, Kluger A, de Leon M, Ferris SH, Convit A, Mittleman M, Cohen J, Rusinek H, De Santi S, George AE (1994) Hippocampal formation size in normal human aging: a correlate of delayed secondary memory performance. Learn Mem 1:45-54.

Guillozet AL, Weintraub S, Mash DC, Mesulam MM (2003) Neurofibrillary tangles, amyloid, and memory in aging and mild cognitive impairment. Arch Neurol 60:729-736.

Hyman BT, Van Horsen GW, Damasio AR, Barnes CL (1984) Alzheimer's disease: cell-specific pathology isolates the hippocampal formation. Science 225:1168-1170.

Insausti R, Juottonen K, Soininen H, Insausti AM, Partanen K, Vainio P, Laakso MP, Pitkanen A (1998) MR volumetric analysis of the human entorhinal, perirhinal, and temporopolar cortices. Am J Neuroradiol 19:659-671.

Jack Jr CR, Petersen RC, Xu Y, O’Brien PC, Smith GE, Ivnik RJ, Boeve BF, Tangalos EG, Kokmen E (2000) Rates of hippocampal atrophy correlate with change in clinical status in aging and AD. Neurology 55:484-489.

Killiany RJ, Gomez-Isla T, Moss M, Kiknis R, Sandor T, Jolesz F, Tanzi R, Jones K, Hyman BT, Albert MS (2000) Use of structural magnetic reso- nance imaging to predict who will get Alzheimer's disease. Ann Neurol 47:430-439.

Kordower JH, Chu Y, Stebbins GT, DeKosky ST, Cochran EJ, Bennett D, Mufson EJ (2001) Loss and atrophy of layer II entorhinal cortex neurons in elderly people with mild cognitive impairment. Ann Neurol 49:202-213.

Laakso MP (2002) Structural imaging in cognitive impairment and the dementias: an update. Curr Opin Neurol 15:415-421.

Laakso MP, Hallikainena M, Hänninen T, Partanen K, Soininen K (2000) Diagnosis of Alzheimer's disease: MRI of the hippocampus vs. delayed recall. Neuropsychologia 38:579-584.

Lindenberger U, Singer T, Baltes PB (2002) Longitudinal selectivity in aging populations: separating mortality-associated versus experimental components in the Berlin Aging Study (BASE). J Gerontol B: Psychol Sci Soc Sci 57:P474-P482.

Oldfield RC (1971) The assessment and analysis of handedness. Neuropsychologia 9:97-113.

Palmer K, Fratiglioni L, Winblad B (2003) What is mild cognitive impairment? Variations in definitions and evolution of nondemented persons with cognitive impairment. Acta Neurol Scand 107:14-20.

Petersen RC, Smith GE, Waring SC, Ivnik RJ, Tangalos EC, Kokmen E (1999) Mild cognitive impairment: clinical characteristics and outcome. Arch Neurol 56:303-308.

Pfefferbaum A, Sullivan EV, Rosenbloom MJ, Mathalon DH, Lim KO (1998) A controlled study of cortical gray matter and ventricular changes in alcoholic men over a 5-year interval. Arch Gen Psychiatry 55:905-912.

Radloff LS (1977) The CES-D scale: a self-report depression scale for research in the general population. Appl Psychol Measurement 1:385-401.

Raz N (2000) Aging of the brain and its impact on cognitive performance: integration of structural and functional findings. In: Handbook of aging and cognition, II (Craik FIM, Salthouse TA, eds), pp 1-90. Mahwah, NJ: Erlbaum.

Raz N, Gunning-Dixon FM, Head DP, Dupuis JH, Acker JD (1998) Neuroanatomical correlates of cognitive aging: evidence from structural MRI. Neuropsychology 12:95-114.

Raz N, Rodrigue KM, Kennedy KM, Dahle C, Head D, Acker JD (2003a) Differential age-related changes in the regional metencephalic volumes in humans: a 5-year follow-up. Neurosci Lett 349:163-166.

Raz N, Rodrigue KM, Kennedy KM, Head D, Gunning-Dixon FM, Acker JD (2003b) Differential aging of the human striatum: longitudinal evidence. Am J Neuroradiol 24:1849-1856.

Raz N, Rodrigue KM, Acker JD (2003c) Hypertension and the brain: vulnerability of the prefrontal regions and executive functions. Behav Neurosci 17:1169-1180.

Raz N, Rodrigue KM, Head D, Kennedy KM, Acker JD (2004a) Differential aging of the medial temporal lobe: a study of a five-year change. Neurology, in press.

Raz N, Gunning-Dixon F, Head D, Rodrigue KM, Williamson A, Acker JD (2004b) Regional volumetry of the cerebral cortex in normal adults: differential aging, sexual dimorphism, and hemispheric asymmetry. Neurobiol Aging, in press.

Resnick SM, Pham DL, Kraut MA, Zonderman AB, Davatzikos C (2003) Longitudinal magnetic resonance imaging studies of older adults: a shrinking brain. J Neurosci 23:3295-3301.

Shrout PE, Fleiss JL (1979) Intraclass correlations: uses in assessing raters reliability. Psychol Bull 86:420-428.

Soininen HS, Partanen K, Pitkanen A, Vainio P, Hanninen T, Hallikainen M, Koivisto K, Riekkinen PJ (1994) Volumetric MRI analysis of the amygdala and the hippocampus in subjects with age-associated memory impairment: correlation to visual and verbal memory. Neurology 44:1660-1668.

Squire LR, Zola-Morgan S (1991) The medial temporal lobe memory system. Science 253:1380-1386.

Strassburger TL, Lee HC, Daly EM, Szczepanik J, Krasuski JS, Mentis MJ, Salerno JA, DeCarli C, Schapiro MB, Alexander GE (1997) Interactive effects of age and hypertension on volumes of brain structures. Stroke 28:1410-1417.

Sullivan E, March L, Mathalon DH, Lim KO, Pfefferbaum A (1995) Agerelated decline in MRI volumes of temporal lobe grey matter but not hippocampus. Neurobiol Aging 16:591-606. 
Thompson PM, Hayashi KM, de Zubicaray G, Janke AL, Rose SE, Semple J, Herman D, Hong MS, Dittmer SS, Doddrell DM, Toga AW (2003) Dynamics of gray matter loss in Alzheimer's disease. J Neurosci 23:994-1005.

Verhaeghen P, Marcoen A, Goosens L (1993) Facts and fiction about memory aging: a quantitative integration of research findings. J Gerontol 48:P157-P171.

Waldstein SR, Katzel LI (2001) Hypertension and cognitive functions. In: Neuropsychology of cardiovascular disease (Waldstein SR, Elias MF, eds), pp 15-36. Mahwah, NJ: LEA.

Wechsler D (1987) Manual for Wechsler memory scale, revised. San Antonio, TX: Psychological Corporation.

Wilson RS, Sullivan M, de Toledo-Morrell L, Stebbins GT, Bennett DA, Mor- rell F (1996) Association of memory and cognition in Alzheimer's disease with volumetric estimates of temporal lobe structures. Neuropsychology 10:459-463.

Woodcock RW, Johnson MB (1989) Manual for Woodcock-Johnson psychoeducational battery, revised. Allen, TX: DLM Teaching Resources.

Woodcock RW, Mather N (1989) Woodcock-Johnson tests of cognitive ability—standard and supplemental batteries: examiner's manual. In: Woodcock-Johnson psychoeducational battery, revised (Woodcock RW, Johnson MB, eds), pp 109-135. Allen, TX: DLM Teaching Resources.

Xu Y, Jack CR, O’Brien PCU, Kokmen E, Smith GE, Ivnik RJ, Boeve BF, Tangalos RG, Peterson RC (2000) Usefulness of MRI measures of entorhinal cortex versus hippocampus in AD. Neurology 54:1760-1767. 